\title{
Three New Triterpene Saponins from the Seeds of Aesculus chinensis
}

\author{
Jing ZHAO, ${ }^{a}$ Xiu-Wei YANG, ${ }^{*}, a$ and Masao HATTORI ${ }^{b}$ \\ National Laboratory of Natural and Biomimetic Drugs, Beijing University, ${ }^{a}$ Beijing 100083, P. R. China and Institute of \\ Natural Medicines, Toyama Medical and Pharmaceutical University, ${ }^{b} 2630$ Sugitani, Toyama 930-0194, Japan. \\ Received November 7, 2000; accepted January 18, 2001
}

\begin{abstract}
Three new triterpenoid saponins were isolated from the seeds of Aesculus chinensis, and characterized as 22-tigloylprotoaescigenin 3- $O$-[ $\beta$-D-glucopyranosyl $(1 \rightarrow 2)][\beta$-D-glucopyranosyl $(1 \rightarrow 4)]-\beta$-D-glucopyranosiduronic acid (escin IVg, 1), 22-angeloylprotoaescigenin 3- $O$ - $[\beta$-D-glucopyranosyl $(1 \rightarrow 2)][\beta$-D-glucopyranosyl $(1 \rightarrow 4)]-\beta$-Dglucopyranosiduronic acid (escin IVh, 2$)$ and 16-angeloyl-21-acetylprotoaescigenin 3- $O$-[ $\beta$-D-glucopyranosyl $(1 \rightarrow$ 2)] [ $\beta$-D-glucopyranosyl $(1 \rightarrow 4)]$ - $\beta$-D-glucopyranosiduronic acid (escin VIb, 3), together with two known compounds, escin IIIa (4) and desacylescin I (5). Their structures were established on the basis of spectroscopic and chemical evidence.
\end{abstract}

Key words Aesculus chinensis; Hippocastanaceae; escin IVg; escin IVh; escin VIb

Escin, ${ }^{1)}$ an anti-inflammatory and anti-edemigenous principle from the seeds of the Chinese horse chestnut tree (Aesculus chinensis Bge., family Hippocastanaceae) consists of a series of analogous pentacyclic triterpenoid oligoglycosides. During our work on the saponin constituents from the seeds, we have isolated and identified escins Ia, Ib, IVc, IVd, IVe and IVf, together with isoescins Ia and Ib. And it was found for the first time that some of them possessed anti-human immunodeficiency virus (HIV)-1 protease activity. ${ }^{2}$ In this paper, we report the isolation and the structure elucidation of five additional saponins, including three new ones.

\section{Results and Discussion}

The crude saponin was obtained from a $90 \%$ ethanolic extract of the seeds of $A$. chinensis as described previously. $\left.{ }^{2}\right)$ After isolation of eight saponins, the remaining fraction was subjected to preparative high-performance liquid chromatography (HPLC) repeatedly affording five saponins. Among them, escin IIIa (4) and desacylescin I (=aesculuside B, 5) were identified by comparison of the spectral data with those reported.,4)

Compound $\mathbf{1}$ was isolated as a white amorphous powder. Matrix-assisted laser desorption ionization-time of flightmass spectrum (MALDI-TOF-MS) showed quasimolecular ion peaks at $m / z 1111[\mathrm{M}+\mathrm{Na}]^{+}$and $1127[\mathrm{M}+\mathrm{K}]^{+}$. Highresolution (HR) negative-ion SI (secondary ion)-MS revealed its molecular formula to be $\mathrm{C}_{53} \mathrm{H}_{84} \mathrm{O}_{23}$. The infrared (IR) spectrum of 1 showed absorption bands at 1710 and $1604 \mathrm{~cm}^{-1}$, characteristic of $\alpha, \beta$-unsaturated ester, and broad bands at 3414 and $1076 \mathrm{~cm}^{-1}$. Alkaline hydrolysis of 1 with $1 \% \mathrm{MeONa}$ liberated desacylescin I (5), which was identified by direct comparison with an authentic sample on HPLC. The proton and carbon-13 nuclear magnetic resonance $\left({ }^{1} \mathrm{H}\right.$ and ${ }^{13} \mathrm{C}$-NMR) spectra showed signals due to the protoaescigenin skeleton [ ${ }^{1} \mathrm{H}-\mathrm{NMR}$ : six quarternary methyl groups at $\delta$ $0.62,0.77,1.26,1.31,1.40,1.82$, and one broad singlet at $\delta 5.42(\mathrm{H}-12)]$, a trisaccharide moiety [ ${ }^{1} \mathrm{H}-\mathrm{NMR}$ : anomeric protons at $\delta 5.55\left(\mathrm{~d}, J=8.0 \mathrm{~Hz}, \mathrm{H}-1^{\prime \prime}\right), 5.21(\mathrm{~d}, J=8.0 \mathrm{~Hz}, \mathrm{H}-$ $\left.1^{\prime \prime \prime}\right), 4.77$ (d, $\left.J=8.0 \mathrm{~Hz}, \mathrm{H}-1^{\prime}\right)$; ${ }^{13} \mathrm{C}-\mathrm{NMR}$ : anomeric carbons at $\left.\delta 104.2\left(\mathrm{C}-1^{\prime}, \mathrm{C}-1^{\prime \prime \prime}\right), 104.1\left(\mathrm{C}-1^{\prime \prime}\right)\right]$ and a tigloyl group [ ${ }^{1} \mathrm{H}-\mathrm{NMR}: \delta 6.98\left(\mathrm{H}-3^{\prime \prime \prime \prime}\right), 1.80\left(\mathrm{Me}-5^{\prime \prime \prime \prime}\right), 1.44\left(\mathrm{Me}-4^{\prime \prime \prime \prime}\right) ;{ }^{13} \mathrm{C}-$ NMR: $\delta 169.3$ (C-1"'"'), 129.4 (C-2'"'), 136.7 (C-3"'"'), 13.9 (C$\left.\left.4^{\prime \prime \prime \prime}\right), 12.2\left(\mathrm{C}-5^{\prime \prime \prime \prime}\right)\right]$. The attachment of a tigloyl group at $\mathrm{C}-22$ was deduced from the heteronuclear multiple-bond coherence (HMBC) experiment, which showed a long-range correlation between $\mathrm{H}-22 \beta(\delta 6.16)$ and a carbonyl carbon ( $\delta 169.3$ ) of a tigloyl group. Furthermore, the trisaccharidic structure was characterized by HMBC correlations between the following pairs: $\mathrm{C}-3(\delta 90.9)$ and $\mathrm{H}-1^{\prime}, \mathrm{C}-2^{\prime}(\delta 79.5)$ and $\mathrm{H}-1^{\prime \prime}$ and $\mathrm{C}-4^{\prime}(\delta 82.7)$ and $\mathrm{H}-1^{\prime \prime \prime}$. Comparison of the ${ }^{13} \mathrm{C}-$ NMR spectral data due to the sugar moiety with those of 5 revealed the identical structure. On the basis of the above evidence, the structure of $\mathbf{1}$ was determined as 22-tigloylprotoaescigenin 3-O-[ $\beta$-D-glucopyranosyl $(1 \rightarrow 2)]$ [ $\beta$-D-glucopyranosyl $(1 \rightarrow 4)]$ - $\beta$-D-glucopyranosiduronic acid, and named escin IVg.

Compound $\mathbf{2}$ was purified as a white amorphous powder. MALDI-TOF-MS showed a quasimolecular ion peak at $\mathrm{m} / \mathrm{z}$ $1111[\mathrm{M}+\mathrm{Na}]^{+}$, while its molecular formula was also suggested to be $\mathrm{C}_{53} \mathrm{H}_{84} \mathrm{O}_{23}$ by $\mathrm{HR}$ negative-ion SI-MS, identical with that of escin IVg (1). Likewise, the IR spectrum showed absorption bands ascribable to an $\alpha, \beta$-unsaturated ester. Alkaline hydrolysis of $\mathbf{2}$ yielded $\mathbf{5}$. The ${ }^{1} \mathrm{H}$ - and ${ }^{13} \mathrm{C}$-NMR spectra of $\mathbf{2}$ were similar to those of $\mathbf{1}$. The only difference between them was the presence of signals due to an angeloyl group [ ${ }^{1} \mathrm{H}-\mathrm{NMR}: \delta 5.85\left(\mathrm{H}-3^{\prime \prime \prime \prime}\right), 1.94\left(\mathrm{Me}-5^{\prime \prime \prime \prime}\right), 2.03(\mathrm{Me}-$ $\left.4^{\prime \prime \prime \prime}\right) ;{ }^{13} \mathrm{C}-\mathrm{NMR}: \delta 169.4\left(\mathrm{C}-1^{\prime \prime \prime \prime}\right), 129.3\left(\mathrm{C}-2^{\prime \prime \prime \prime}\right), 136.5\left(\mathrm{C}-3^{\prime \prime \prime \prime}\right)$, $\left.15.8\left(\mathrm{C}-4^{\prime \prime \prime \prime}\right), 20.8\left(\mathrm{C}-5^{\prime \prime \prime \prime}\right)\right]$ instead of those of a tigloyl group. The HMBC experiment confirmed the same acylation position and the identical trisaccharide moiety. Therefore, $\mathbf{2}$ was established as 22-angeloylprotoaescigenin 3- $O$-[ $\beta$-D-glucopyranosyl $(1 \rightarrow 2)$ ] [ $\beta$-D-glucopyranosyl $(1 \rightarrow 4)]-\beta$-D-glucopyranosiduronic acid, and named escin IVh. Escins IVg and IVh are a pair of geometrical isomers.

Compound 3 was obtained as a white amorphous powder. MALDI-TOF-MS showed the quasimolecular ion peak at $\mathrm{m} / \mathrm{z}$ $1153[\mathrm{M}+\mathrm{Na}]^{+}$. Its high-resolution positive-ion MALDITOF-MS revealed its molecular formula to be $\mathrm{C}_{55} \mathrm{H}_{86} \mathrm{O}_{24}$. It suggested that $\mathbf{3}$ has the same molecular composition as previously isolated escin Ia, which has been determined by a single-crystal X-ray analysis. ${ }^{2)}$ The ${ }^{1} \mathrm{H}$ - and ${ }^{13} \mathrm{C}-\mathrm{NMR}$ spectra exhibited signals assignable to the protoaescigenin skeleton, an angeloyl group [ ${ }^{1} \mathrm{H}-\mathrm{NMR}: \delta 5.87\left(\mathrm{H}-3^{\prime \prime \prime \prime}\right), 1.90\left(\mathrm{Me}-5^{\prime \prime \prime \prime}\right)$, $1.98\left(\mathrm{Me}-4^{\prime \prime \prime \prime}\right) ;{ }^{13} \mathrm{C}-\mathrm{NMR}: \delta 168.3\left(\mathrm{C}-1^{\prime \prime \prime \prime}\right), 129.2\left(\mathrm{C}-2^{\prime \prime \prime \prime}\right)$, $\left.136.0\left(\mathrm{C}-3^{\prime \prime \prime \prime}\right), 15.9\left(\mathrm{C}-4^{\prime \prime \prime \prime}\right), 21.0\left(\mathrm{C}-5^{\prime \prime \prime \prime}\right)\right]$, an acetyl group [ ${ }^{1} \mathrm{H}-\mathrm{NMR}: \delta 2.50$ (Me-OAc); ${ }^{13} \mathrm{C}-\mathrm{NMR}: \delta 170.0\left(\mathrm{C}-1^{\prime \prime \prime \prime \prime}\right)$, 


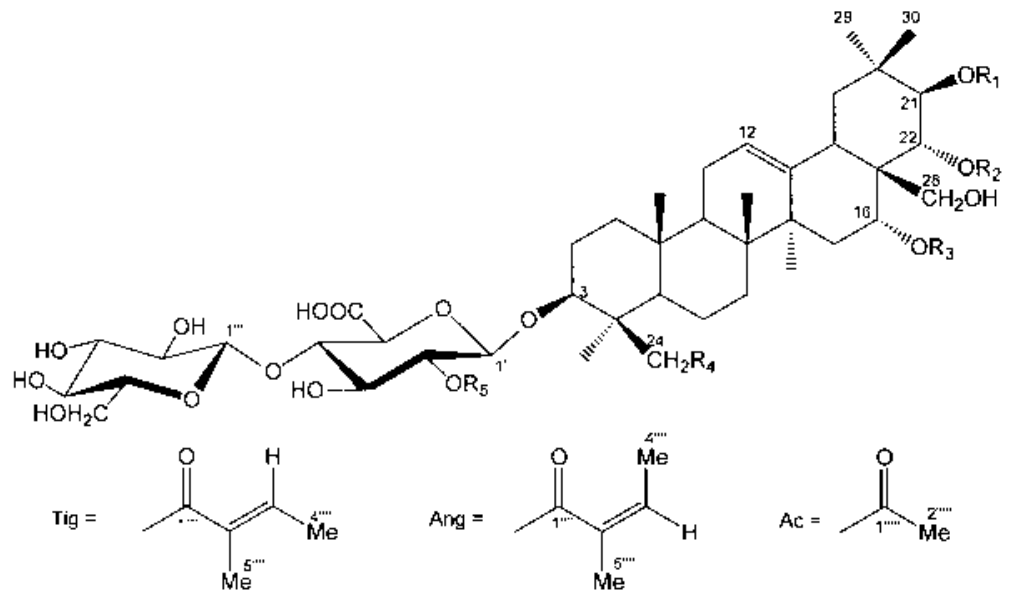

$$
\begin{aligned}
& \mathrm{Glc}=\beta-\mathrm{D}-\mathrm{glucp} \text { pyranosyl } \\
& \mathrm{Gal}=\beta-\mathrm{D}-\mathrm{galactopy} \text { ranosyl }
\end{aligned}
$$

$\begin{array}{cccccc} & \mathbf{R}_{\mathbf{1}} & \mathbf{R}_{\mathbf{2}} & \mathbf{R}_{\mathbf{3}} & \mathbf{R}_{\mathbf{4}} & \mathbf{R}_{5} \\ 1 & \mathbf{H} & \mathrm{Tig} & \mathrm{H} & \mathrm{OH} & \text { Glc } \\ 2 & \mathrm{H} & \mathrm{Ang} & \mathrm{H} & \mathrm{OH} & \text { Gic } \\ 3 & \mathrm{AC} & \mathrm{H} & \mathrm{Ang} & \mathrm{OH} & \text { Glc } \\ 4 & \text { Tig } & \mathrm{AC} & \mathrm{H} & \mathrm{H} & \text { Gal } \\ 5 & \mathrm{H} & \mathrm{H} & \mathrm{H} & \mathrm{OH} & \text { Glc }\end{array}$

Table 1. ${ }^{13} \mathrm{C}-\mathrm{NMR}$ Spectral Data for Escins IVg (1), IVh (2), and VIb (3) $(\delta \text { Values })^{a)}$

\begin{tabular}{rrrrrrrr}
\hline \hline C & \multicolumn{1}{c}{$\mathbf{1}$} & $\mathbf{2}$ & $\mathbf{3}$ & $\mathrm{C}$ & $\mathbf{1}$ & $\mathbf{2}$ & \multicolumn{1}{c}{$\mathbf{3}$} \\
\hline 1 & 38.3 & 38.3 & 38.4 & 29 & 30.1 & 30.1 & 30.0 \\
2 & 26.2 & 26.2 & 26.4 & 30 & 19.2 & 19.2 & 20.1 \\
3 & 90.9 & 90.9 & 91.2 & $1^{\prime}$ & 104.2 & 104.2 & 104.7 \\
4 & 43.4 & 43.4 & 43.6 & $2^{\prime}$ & 79.5 & 79.6 & 79.4 \\
5 & 55.9 & 55.9 & 56.0 & $3^{\prime}$ & 76.5 & 76.4 & 76.7 \\
6 & 18.4 & 18.3 & 18.4 & $4^{\prime}$ & 82.7 & 82.7 & 82.4 \\
7 & 33.0 & 33.0 & 33.1 & $5^{\prime}$ & 75.4 & 75.5 & 75.8 \\
8 & 39.7 & 39.7 & 39.9 & $6^{\prime}$ & 175.0 & 175.1 & 172.9 \\
9 & 46.5 & 46.5 & 46.7 & $1^{\prime \prime}$ & 104.1 & 103.9 & 104.1 \\
10 & 36.2 & 36.2 & 36.3 & $2^{\prime \prime}$ & 75.4 & 75.5 & 75.6 \\
11 & 23.9 & 23.9 & 23.9 & $3^{\prime \prime}$ & 78.1 & 78.2 & 78.2 \\
12 & 123.2 & 123.0 & 123.2 & $4^{\prime \prime}$ & 69.6 & 69.6 & 69.8 \\
13 & 143.1 & 143.1 & 141.8 & $5^{\prime \prime}$ & 77.4 & 77.4 & 78.0 \\
14 & 40.2 & 40.2 & 41.3 & $6^{\prime \prime}$ & 61.4 & 61.4 & 61.6 \\
15 & 34.6 & 34.6 & 30.9 & $1^{\prime \prime \prime}$ & 104.2 & 104.3 & 104.7 \\
16 & 68.9 & 69.0 & 71.5 & $2^{\prime \prime \prime}$ & 74.8 & 74.8 & 73.9 \\
17 & 48.0 & 47.7 & 47.6 & $3^{\prime \prime \prime}$ & 77.9 & 78.2 & 78.4 \\
18 & 41.4 & 41.4 & 39.9 & $4^{\prime \prime \prime}$ & 71.2 & 71.1 & 71.5 \\
19 & 47.5 & 47.5 & 47.2 & $5^{\prime \prime \prime}$ & 77.8 & 77.8 & 77.9 \\
20 & 36.8 & 36.9 & 36.0 & $6^{\prime \prime \prime}$ & 61.9 & 61.9 & 62.2 \\
21 & 76.5 & 76.6 & 79.8 & $1^{\prime \prime \prime \prime}$ & 169.3 & 169.4 & 168.3 \\
22 & 77.4 & 77.1 & 71.0 & $2^{\prime \prime \prime \prime}$ & 129.4 & 129.3 & 129.2 \\
23 & 22.3 & 22.3 & 22.4 & $3^{\prime \prime \prime \prime}$ & 136.7 & 136.5 & 136.0 \\
24 & 63.1 & 63.1 & 63.2 & $4^{\prime \prime \prime \prime}$ & 13.9 & 15.8 & 15.9 \\
25 & 15.4 & 15.4 & 15.5 & $5^{\prime \prime \prime \prime}$ & 12.2 & 20.8 & 21.0 \\
26 & 16.5 & 16.5 & 16.6 & $1^{\prime \prime \prime \prime \prime \prime \prime}$ & & & 170.0 \\
27 & 27.3 & 27.4 & 27.0 & $2^{\prime \prime \prime \prime \prime \prime \prime}$ & & & 22.2 \\
28 & 63.9 & 64.1 & 64.8 & & & & \\
\hline & & & & & & &
\end{tabular}

a) Measured in pyridine- $d_{5}$.

$\left.22.2\left(\mathrm{C}-2^{\prime \prime \prime \prime \prime}\right)\right]$ and a trisaccharide moiety $\left[{ }^{1} \mathrm{H}-\mathrm{NMR}: \delta 4.79\right.$ $\left(\mathrm{d}, J=7.5 \mathrm{~Hz}, \mathrm{H}-1^{\prime}\right), 5.33\left(\mathrm{~d}, J=7.5 \mathrm{~Hz}, \mathrm{H}-1^{\prime \prime \prime}\right)$ and $5.60(\mathrm{~d}$, $\left.J=7.5 \mathrm{~Hz}, \mathrm{H}-1^{\prime \prime}\right) ;{ }^{13} \mathrm{C}-\mathrm{NMR}: \delta 104.7\left(\mathrm{C}-1^{\prime}, \mathrm{C}-1^{\prime \prime \prime}\right)$ and 104.1 $\left(\mathrm{C}-1^{\prime \prime}\right)$ ]. Compared with the protoaescigenin skeleton of $\mathbf{5}$, however, C-16 shifted to the downfield by $3.8 \mathrm{ppm}$, while C-
15 shifted to the upfield by $3.2 \mathrm{ppm}$. HMBC correlation between signals of $\mathrm{H}-16 \beta(\delta 5.90)$ and a carbonyl carbon ( $\delta 168.3)$ of an angeloyl group indicated that the angeloyl group is attached to C-16. An acetyl group was determined to attach to C-21 on the basis of the deshielding of $1.3 \mathrm{ppm}$ for $\mathrm{C}-21$ and the shielding of $6.0 \mathrm{ppm}$ for $\mathrm{C}-22$, relative to those of 5. Moreover, a correlation between H-21 $(\delta 5.95)$ and $\mathrm{Me}-\mathrm{OAc}(\delta 2.50)$ in the nuclear Overhouser effect spectrometry (NOESY) spectrum also inferred the structure. The sugar moiety of $\mathbf{3}$ was determined to be identical with that of 5 after alkaline hydrolysis. Consequently, the structure of $\mathbf{3}$ was concluded to be 16-angeloyl-21-acetylprotoaescigenin 3- $O$-[ $\beta$-D-glucopyranosyl $(1 \rightarrow 2)]$ [ $\beta$-D-glucopyranosyl $(1 \rightarrow$ 4)]- $\beta$-D-glucopyranosiduronic acid, and named escin VIb. It was the first C-16 acylated compound from escins.

It should be noted that since all the above compounds were detected by HPLC analysis of the ethanolic extract, they are not artifacts produced during the isolation process. Recently, Zhang et al. ${ }^{5)}$ reported eight new triterpene saponins, aesculiosides $\mathrm{A}-\mathrm{H}$ from the seeds of the same plant, but their structures were different in acylated positions from the saponins isolated at the present time.

\section{Experimental}

General IR: $\mathrm{KBr}$ discs, Perkin-Elmer 983 model; NMR: Varian INOVA-500 Spectrometer operating at $500 \mathrm{MHz}$ for ${ }^{1} \mathrm{H}$ and $125 \mathrm{MHz}$ for ${ }^{13} \mathrm{C}$ including ${ }^{1} \mathrm{H}-{ }^{1} \mathrm{H}$ correlation spectroscopy (COSY), ${ }^{1} \mathrm{H}$-detected multiple quantum coherence (HMQC), heteronuclear multiple bond correlation (HMBC) and NOESY. Chemical shifts are given in $\delta$ relative to TMS as internal standard. MALDI-TOF-MS: BIFLEX III (Bruker). HR-SI-MS: APEX II FT-ICRMS (Bruker Daltonics). For preparative HPLC (pump: P2000; detector: UV3000 and software: PC1000. Thermo Separation Products, U.S.A.), an octadecyl sil (ODS) column $\left[10 \mu \mathrm{C}_{18}\right.$ (2), $250 \times 21.2 \mathrm{~mm}$, Phenomenex, U.S.A.] column was used. For HPLC analysis, an ODS column [LUNA $5 \mu \mathrm{C}_{18}$ (2), $250 \times 4.60 \mathrm{~mm}$, Phenomenex, U.S.A.] was used.

Plant Material The seeds of Aesculus chinensis Bge. were collected at Lueyang County, Shaanxi Province, P. R. China, in September 1997. The voucher specimens are deposited at the National Laboratory of Natural and 
Biomimetic Drugs, Peking University.

Extraction and Isolation of Compounds The seeds of $A$. chinensis were extracted as described previously. ${ }^{2)}$ Further chromatography of the $70 \%$ EtOH fraction on prep. HPLC [MeCN-0.5\% AcOH aq. $(7: 3$ and $6: 4, \mathrm{v} / \mathrm{v})]$ yielded escins IVg (1,32 mg), IVh (2, 35 mg), VIb (3, $41 \mathrm{mg})$, IIIa (4, $50 \mathrm{mg})$ and desacylescin I (5, $75 \mathrm{mg})$.

Alkaline Hydrolysis of Saponins 1-3 The saponin (10 mg of $\mathbf{1}, \mathbf{2}$ or 3 , respectively) was added to a $\mathrm{MeOH}$ solution $(10 \mathrm{ml})$ of $\mathrm{MeONa}(1 \mathrm{mg})$. The mixture was stirred at room temperature for $8 \mathrm{~h}$ and then neutralized with Dowex $50 \mathrm{~W} \times 8\left(\mathrm{H}^{+}\right.$form $)$. After removal of the solvent in vacuo, the residue was dissolved in $\mathrm{MeOH}(1 \mathrm{ml})$ and analyzed by HPLC [solvent, $\mathrm{MeOH}-1 \%$ aqueous phosphoric acid $(7: 3)$; flow rate, $0.5 \mathrm{ml} / \mathrm{min}$; wavelength, $220 \mathrm{~nm}$ ].

Escin IVg (1): White amorphous powder. $[\alpha]_{\mathrm{D}}^{25}=-25.0^{\circ}(\mathrm{MeOH} ; c=$ 1.00). IR (KBr) $v_{\max }: 3423,2922,1710,1604,1407,1273,1076,1040$, $617 \mathrm{~cm}^{-1}$. MALDI-TOF-MS $m / z 1111[\mathrm{M}+\mathrm{Na}]^{+}, 1127[\mathrm{M}+\mathrm{K}]^{+}$. HR-SIMS (negative-ion mode) $\mathrm{m} / z$ : 1087.5291 , Calcd for $\mathrm{C}_{53} \mathrm{H}_{83} \mathrm{O}_{23}[\mathrm{M}-\mathrm{H}]^{-}$: 1087.5331. ${ }^{1} \mathrm{H}-\mathrm{NMR}\left(500 \mathrm{MHz}\right.$, pyridine- $\left.d_{5}\right) \delta: 0.62\left(3 \mathrm{H}, \mathrm{s}, \mathrm{H}_{3}-25\right), 0.65$ $(1 \mathrm{H}, \mathrm{d}, J=12.5 \mathrm{~Hz}, \mathrm{H}-5), 0.77\left(3 \mathrm{H}, \mathrm{s}, \mathrm{H}_{3}-26\right), 1.21\left(2 \mathrm{H}, \mathrm{m}, \mathrm{H}_{2}-7\right), 1.26(3 \mathrm{H}$, $\left.\mathrm{s}, \mathrm{H}_{3}-23\right), 1.31\left(3 \mathrm{H}, \mathrm{s}, \mathrm{H}_{3}-29\right), 1.40\left(3 \mathrm{H}, \mathrm{s}, \mathrm{H}_{3}-30\right), 1.44(1 \mathrm{H}, \mathrm{d}, J=7.0 \mathrm{~Hz}$, $\left.\mathrm{H}_{3}-4^{\prime \prime \prime \prime}\right), 1.55(1 \mathrm{H}, \mathrm{d}, J=14.5 \mathrm{~Hz}, \mathrm{H}-9), 1.80\left(3 \mathrm{H}, \mathrm{s}, \mathrm{H}_{3}-5^{\prime \prime \prime \prime}\right), 1.82\left(3 \mathrm{H}, \mathrm{s}, \mathrm{H}_{3}-\right.$ 27), $3.02\left(1 \mathrm{H}, \mathrm{t}, J=13.5 \mathrm{~Hz}, \mathrm{H}_{\mathrm{b}}-19\right), 3.11(1 \mathrm{H}, \mathrm{d}, J=13.5 \mathrm{~Hz}, \mathrm{H}-18), 3.28$ $\left(1 \mathrm{H}, \mathrm{d}, J=11.5 \mathrm{~Hz}, \mathrm{H}_{\mathrm{a}}-24\right), 3.30(1 \mathrm{H}$, dd-like, $\mathrm{H}-3 \alpha), 3.34(1 \mathrm{H}, \mathrm{d}$, $\left.J=11.0 \mathrm{~Hz}, \mathrm{H}_{\mathrm{a}}-28\right), 3.60\left(1 \mathrm{H}, \mathrm{d}, J=11.0 \mathrm{~Hz}, \mathrm{H}_{\mathrm{b}}-28\right), 4.21(1 \mathrm{H}, \mathrm{d}, J=11.5 \mathrm{~Hz}$, $\left.\mathrm{H}_{\mathrm{b}}-24\right), 4.47(1 \mathrm{H}, \mathrm{br} \mathrm{s}, \mathrm{H}-16 \beta), 4.77\left(1 \mathrm{H}, \mathrm{d}, J=8.0 \mathrm{~Hz}, \mathrm{H}-1^{\prime}\right), 5.07(1 \mathrm{H}, \mathrm{d}$, $J=9.5 \mathrm{~Hz}, \mathrm{H}-21 \alpha), 5.21\left(1 \mathrm{H}, \mathrm{d}, J=8.0 \mathrm{~Hz}, \mathrm{H}-1^{\prime \prime \prime}\right), 5.42(1 \mathrm{H}, \mathrm{br} \mathrm{s}, \mathrm{H}-12), 5.55$ $\left(1 \mathrm{H}, \mathrm{d}, J=8.0 \mathrm{~Hz}, \mathrm{H}-1^{\prime \prime}\right), 6.16(1 \mathrm{H}, \mathrm{d}, J=9.5 \mathrm{~Hz}, \mathrm{H}-22 \beta), 6.99(1 \mathrm{H}, \mathrm{dq}-\mathrm{like}$, $\left.\mathrm{H}-3^{\prime \prime \prime \prime}\right)$. ${ }^{13} \mathrm{C}-\mathrm{NMR}$ spectral data are given in Table 1 .

Escin IVh (2): White amorphous powder. $[\alpha]_{\mathrm{D}}^{25}=-60^{\circ}(\mathrm{MeOH} ; c=1.05)$. IR (KBr) $v_{\max }$ : 3409, 2922, 1713, 1609, 1411, 1242, 1164, 1074, 1040, $612 \mathrm{~cm}^{-1}$. MALDI-TOF-MS $\mathrm{m} / z 1111[\mathrm{M}+\mathrm{Na}]^{+}$. HR-SI-MS (negative ion mode) $m / z: 1087.5308$, Calcd for $\mathrm{C}_{53} \mathrm{H}_{83} \mathrm{O}_{23}[\mathrm{M}-\mathrm{H}]^{-}:$: 1087.5331. ${ }^{1} \mathrm{H}-\mathrm{NMR}$ $\left(500 \mathrm{MHz}\right.$, pyridine- $\left.d_{5}\right) \delta: 0.60\left(3 \mathrm{H}, \mathrm{s}, \mathrm{H}_{3}-25\right), 0.77\left(3 \mathrm{H}, \mathrm{s}, \mathrm{H}_{3}-26\right), 1.24$ (3H, s, $\left.\mathrm{H}_{3}-23\right), 1.27$ (3H, s, $\left.\mathrm{H}_{3}-29\right), 1.34\left(1 \mathrm{H}, \mathrm{m}, \mathrm{H}_{\mathrm{a}}-19\right), 1.38\left(3 \mathrm{H}, \mathrm{s}, \mathrm{H}_{3}-30\right)$, $1.56(1 \mathrm{H}, \mathrm{d}, J=14.5 \mathrm{~Hz}, \mathrm{H}-9), 1.80\left(3 \mathrm{H}, \mathrm{s}, \mathrm{H}_{3}-27\right), 1.94\left(3 \mathrm{H}, \mathrm{s}, \mathrm{H}_{3}-5^{\prime \prime \prime}\right), 2.03$ $\left(1 \mathrm{H}, \mathrm{d}, J=6.0 \mathrm{~Hz}, \mathrm{H}_{3}-4^{\prime \prime \prime \prime}\right), 3.02\left(1 \mathrm{H}, \mathrm{t}, J=11.0 \mathrm{~Hz}, \mathrm{H}_{\mathrm{b}}-19\right), 3.07(1 \mathrm{H}, \mathrm{d}$, $J=11.0 \mathrm{~Hz}, \mathrm{H}-18), 3.27\left(1 \mathrm{H}, \mathrm{d}-\mathrm{like}, \mathrm{H}_{\mathrm{a}}-24\right), 3.28(1 \mathrm{H}, \mathrm{br} \mathrm{s}, \mathrm{H}-3 \alpha), 3.38(1 \mathrm{H}$, d, $\left.J=9.5 \mathrm{~Hz}, \mathrm{H}_{\mathrm{a}}-28\right), 3.61\left(1 \mathrm{H}, \mathrm{d}, J=9.5 \mathrm{~Hz}, \mathrm{H}_{\mathrm{b}}-28\right), 4.40(1 \mathrm{H}, \mathrm{d}, J=11.5 \mathrm{~Hz}$,
$\left.\mathrm{H}_{\mathrm{b}}-24\right), 4.48(1 \mathrm{H}, \mathrm{brs}, \mathrm{H}-16 \beta), 4.73\left(1 \mathrm{H}, \mathrm{d}, J=8.0 \mathrm{~Hz}, \mathrm{H}-1^{\prime}\right), 5.02(1 \mathrm{H}, \mathrm{d}$, $J=9.0 \mathrm{~Hz}, \mathrm{H}-21 \alpha), 5.21\left(1 \mathrm{H}, \mathrm{d}, J=8.0 \mathrm{~Hz}, \mathrm{H}-1^{\prime \prime \prime}\right), 5.42(1 \mathrm{H}, \mathrm{br} \mathrm{s}, \mathrm{H}-12), 5.51$ $\left(1 \mathrm{H}, \mathrm{d}, J=8.0 \mathrm{~Hz}, \mathrm{H}-1^{\prime \prime}\right), 5.85\left(1 \mathrm{H}, \mathrm{dq}-1 \mathrm{ike}, \mathrm{H}-3^{\prime \prime \prime}\right), 6.16(1 \mathrm{H}, \mathrm{d}, J=9.0 \mathrm{~Hz}$, $\mathrm{H}-22 \beta) .{ }^{13} \mathrm{C}-\mathrm{NMR}$ spectral data are given in Table 1.

Escin VIb (3): White amorphous powder. $[\alpha]_{\mathrm{D}}^{25}=-55^{\circ}(\mathrm{MeOH} ; c=1.00)$. IR (KBr) $v_{\max }: 3408,2923,1711,1570,1421,1260,1075,1044,659 \mathrm{~cm}^{-1}$. MALDI-TOF-MS $m / z 1153[\mathrm{M}+\mathrm{Na}]^{+}$. HR MALDI-TOF-MS (positive ion mode) $m / z$ : 1153.5432, Calcd for $\mathrm{C}_{55} \mathrm{H}_{86} \mathrm{O}_{24} \mathrm{Na}[\mathrm{M}+\mathrm{Na}]^{+}: 1153.5401 .{ }^{1} \mathrm{H}-$ NMR $\left(500 \mathrm{MHz}\right.$, pyridine- $\left.d_{5}\right) \delta: 0.65\left(3 \mathrm{H}, \mathrm{s}, \mathrm{H}_{3}-25\right), 0.65\left(3 \mathrm{H}, \mathrm{s}, \mathrm{H}_{3}-26\right)$, $1.10\left(3 \mathrm{H}, \mathrm{s}, \mathrm{H}_{3}-29\right), 1.22\left(1 \mathrm{H}, \mathrm{d}, J=13.5 \mathrm{~Hz}, \mathrm{H}_{\mathrm{a}}-19\right), 1.26\left(3 \mathrm{H}, \mathrm{s}, \mathrm{H}_{3}-23\right)$, $1.26\left(3 \mathrm{H}, \mathrm{s}, \mathrm{H}_{3}-30\right), 1.47\left(3 \mathrm{H}, \mathrm{s}, \mathrm{H}_{3}-27\right), 1.90\left(3 \mathrm{H}, \mathrm{s}, \mathrm{H}_{3}-5^{\prime \prime \prime \prime}\right), 1.98(3 \mathrm{H}, \mathrm{d}$, $\left.J=7.0 \mathrm{~Hz}, \mathrm{H}_{3}-4^{\prime \prime \prime \prime}\right), 2.50(3 \mathrm{H}, \mathrm{s}, \mathrm{OAc}), 2.69\left(1 \mathrm{H}, \mathrm{t}, J=10.0 \mathrm{~Hz}, \mathrm{H}_{\mathrm{b}}-19\right), 2.94$ $(1 \mathrm{H}, \mathrm{d}, J=13.0 \mathrm{~Hz}, \mathrm{H}-18), 3.24(1 \mathrm{H}, \mathrm{dd}-\mathrm{like}, \mathrm{H}-3 \alpha), 4.51(1 \mathrm{H}, \mathrm{d}$, $J=10.0 \mathrm{~Hz}, \mathrm{H}-22 \beta), 4.79(1 \mathrm{H}, \mathrm{d}, J=7.5 \mathrm{~Hz}, \mathrm{H}-19), 5.33(1 \mathrm{H}, \mathrm{d}, J=7.5 \mathrm{~Hz}$, H-1"'), $5.60\left(1 \mathrm{H}, \mathrm{d}, J=7.5 \mathrm{~Hz}, \mathrm{H}-1^{\prime \prime}\right), 5.57(1 \mathrm{H}, \mathrm{br} \mathrm{s}, \mathrm{H}-12), 5.87(1 \mathrm{H}, \mathrm{dq}-$ like, H-3"'"'), $5.90(1 \mathrm{H}, \mathrm{d}, J=4.5 \mathrm{~Hz}, \mathrm{H}-16 \beta), 5.95(1 \mathrm{H}, \mathrm{d}, J=10.0 \mathrm{~Hz}, \mathrm{H}-$ $21 \alpha) .{ }^{13} \mathrm{C}$-NMR spectral data are given in Table 1.

Escin IIIa (4): White amorphous powder. $[\alpha]_{\mathrm{D}}^{25}=-53.3^{\circ}(\mathrm{MeOH} ; c=$ 0.90). IR (KBr) $v_{\text {max }}: 3423,2922,1709,1609,1382,1272,1074,600 \mathrm{~cm}^{-1}$. MALDI-TOF-MS $m / z: 1137[\mathrm{M}+\mathrm{Na}]^{+}$.

Desacylescin I (5): Colorless fine crystals, mp $260-262{ }^{\circ} \mathrm{C} .[\alpha]_{\mathrm{D}}^{25}$ $=-33.9^{\circ}(\mathrm{MeOH} ; c=1.15)$. IR $(\mathrm{KBr}) v_{\max }: 3400,2940,2328,1725,1607$, $1413,1376,1260,1159,1074,1029,904,858,798,638 \mathrm{~cm}^{-1}$. MALDITOF-MS $m / z 1029[\mathrm{M}+\mathrm{Na}]^{+}, 1045[\mathrm{M}+\mathrm{K}]^{+}$.

Acknowledgments This work was financially supported by the National Natural Science Foundation of China (29972004).

\section{References}

1) Zhou M. Z., Wang Y. Y., Chinese New Drugs J., 5, 438 (1996).

2) Yang X. W., Zhao J., Cui Y. X., Liu X. H., Ma C. M., Hattori M., Zhang L. H., J. Nat. Prod., 62, 1510 (1999).

3) Singh B., Agrawal P. K., Thakur R. S., J. Nat. Prod., 50, 781 (1987).

4) Yoshikawa M., Murakami T., Matsuda H., Yamahara J., Murakami N., Kitagawa I., Chem. Pharm. Bull., 44, 1454 (1996).

5) Zhang Z., Koike K., Jia Z., Nikaido T., Guo D., Zheng J., Chem. Pharm. Bull., 47, 1515-1520 (1999). 